\title{
Pruebas de ecotoxicidad para establecer el potencial genotóxico del hipoclorito de sodio, mediante bulbos de cebolla Allium cepa $L$ y semillas de lechuga Lactuca sativa L como bioindicadores
}

\author{
Ricardo Restrepo Manrique \\ Esp. en Química Ambiental, \\ Universidad Industrial de Santander \\ Docente, Investigador Grupo GIA, \\ Universidad Santo Tomás USTA \\ Bucaramanga, Colombia \\ restrepo.manrique@gmail.com
}

\author{
Marlyn Catalina Ortiz Villamizar \\ Estudiante de Química Ambiental, \\ Universidad Santo Tomás USTA \\ Bucaramanga, Colombia \\ catalina.ortiz12@gmail.com
}

\author{
Diana Reyes Quesada \\ Estudiante de Química Ambiental, \\ Universidad Santo Tomás USTA \\ Bucaramanga, Colombia \\ reyesquesada.diana@gmail.com
}

Resumen-Se realizan pruebas de toxicidad mediante bulbos de cebolla (Allium cepa) y se confrontan los resultados con la prueba en semillas de lechuga (Lactuca sativa), bioindicador reconocido internacionalmente según la OECD. Las pruebas se montaron en hipoclorito de sodio $(\mathrm{NaClO})$ al $5 \%$, concentración comercial para uso doméstico. El objetivo principal de este trabajo es el de establecer el nivel de toxicidad de este producto y confrontarlo con las concentraciones de uso doméstico, siguiendo las recomendaciones del fabricante. Las plantas utilizadas como bioindicadoras permiten establecer las concentraciones de inhibición (CI50) y la genotoxicidad, a partir de las células meristemáticas de la cebolla (Allium cepa).

Los resultados obtenidos son coincidentes, el hipoclorito de sodio es tóxico para ambas plantas, obteniéndose una $\mathrm{Cl} 50$ de 17,27 mg NaClO/I para la lechuga (Lactuca sativa) e inferior a $5 \mathrm{mg} \mathrm{NaClO/I} \mathrm{para} \mathrm{cebolla} \mathrm{(Allium}$ cepa), concentraciones muy inferiores a las que se usa en las dosificaciones domésticas. Se manifestaron aberraciones cromosómicas en células meristémicas de Alluim cepa como anafase con puente, rompimiento de cromosomas en metafase, malformación de células con núcleos periféricos y telofase con pérdida de cromosomas, todas estas reconocidas internacionalmente como aberraciones generadas por sustancias químicas tóxicas. Así mismo, se establece la especie Alliun cepa es más sensible que Lactuca sativa, y es viable realizar los montajes con cebolla a nivel de laboratorio. Sus resultados se constituyen como complemento en el diagnóstico genotóxico de aguas naturales o en ecosistemas receptores de vertimientos.

Palabras clave- Bioindicadores, pruebas de toxicidad, hipoclorito de sodio, genotoxicidad, Allium cepa, Lactuca sativa.

\begin{abstract}
The toxicity test that we made use bulbs of onion (Allium cepa) and compared the results with the test of lettuce seeds (Lactuca sativa), like a bioindicator recognized by internationally to the OECD. The tests were made on sodium hypochlorite ( $\mathrm{NaClO}$ ) to $5 \%$; this concentration is for the domestic use. The main objective for this work is establish the level of toxicity to that product y compared with the concentrations for the domestic use, following the manufacturer's recommendations. The plants used like bioindicator allow establish the inhibition concentrations (IC50) and genotoxicity, from meristematics cells of onion (Allium cepa)
\end{abstract}

The results obtain are consistent, the sodium hypochlorite is toxic to both plants, obtained a IC50 of $17.27 \mathrm{mg} \mathrm{NaClO} / \mathrm{I}$ for lettuce (Lactuca sativa) and less $5 \mathrm{mg} \mathrm{NaClO/I} \mathrm{for} \mathrm{onion}$ (Allium cepa), the concentrations are much lower than the people use in their houses. The chromosomal aberrations were showed in meristem cells of Allium cepa as bridge anaphase, chromosome breakage in metaphase, malformation of cells with eccentric nuclei and telophase with chromosome loss, those chromosomal aberrations are recognized internationally like aberrations generated by toxic chemical substances. Thus concludes that specie Allium cepa is more sensitive than Lactuca sativa, and it is feasible to conduct experiments in the laboratory with onion. Their results are complement to genotoxic diagnosis for the natural waters or recipient ecosystems of discharge.

Keywords- Bioindicator, toxicity tests, sodium hypochlorite, genotoxicity, Allium cepa, Lactuca sativa

\section{INTRODUCCIÓN}

La contaminación de los recursos naturales cada día aporta más compuestos químicos en los 
cuerpos de agua, asociados a las actividades industriales, agropecuaria, porcicultura, avicultura, hasta los mismos municipios que vierten los residuos sólidos y líquidos sin la total remoción de los elementos contaminantes.

Un agua clara y potable es una necesidad humana básica; sin embargo, el acceso a ella es aún una gran dificultad para muchas comunidades de países en desarrollo. La contaminación de agua por organismos patógenos constituye todavía una fuente de enfermedades importante en estos países, un gran número de poblaciones se enfrenta, además, con una contaminación química creciente proveniente del uso de agroquímicos, actividades industriales y fuentes domésticas. A pesar de este panorama, en las décadas pasadas se ha trabajado intensamente en el desarrollo y validación de diversos métodos de diagnóstico fisicoquímico del agua, y varias técnicas han sido adaptadas para uso a nivel de comunidad de manera sustentable.

Por acción biológica, física o química, estos compuestos se complejizan en el medio y muchos de estos se transforman en componentes más tóxicos que sus generadores, constituyéndose en un riesgo ambiental y de salubridad cuando estos recursos son aprovechados por la comunidad. La analítica química no ha desarrollado aún las marchas y procedimientos para detectar la presencia de estos componentes en el medio, es acá donde la toxicología ambiental, a través de las pruebas de ecotoxicidad, permite dilucidar cualitativamente la presencia de estas sustancias nocivas, se advierte el riesgo de utilizar el recurso para consumo doméstico.

\section{TOXICOLOGÍA AMBIENTAL Y ECOTOXICOLOGÍA}

Los términos Toxicología Ambiental y Ecotoxicología describen los estudios de los efectos adversos sobre los organismos vivos que originan las sustancias químicas cuando entran en contacto con el medioambiente. [1]. Existe una tendencia a denominar Toxicología Ambiental sólo a los estudios sobre los efectos directos de los químicos medioambientales en los humanos y Ecotoxicología al estudio de los efectos de los químicos en los ecosistemas y sus componentes no humanos. Sin embargo, los humanos no estamos aislados del medioambiente natural sino que somos una parte integral de él y él es modificado por nuestras acciones directas o indirectas. [2].

La Ecotoxicología es una disciplina joven y fue definida por primera vez en 1974, hace poco más de 30 años, por René Truhaut. En la concepción como rama de la Toxicología intenta combinar la Ecología y la Toxicología. [2]. La Ecotoxicología pretende no sólo evaluar el impacto de los químicos en el hombre sino también en las poblaciones y los ecosistemas. [1].

Una definición más completa de Ecotoxicología fue propuesta por Kruijf en 1988 [3] cuando planteó que es el campo de estudio que integra los efectos ecológicos y toxicológicos de los contaminantes químicos en las poblaciones, comunidades y ecosistemas con el destino (transporte, transformación y degradación) de tales contaminantes en el ambiente.

La mayor parte de la información toxicológica disponible en el mundo está relacionada con los efectos directos de las sustancias químicas en el humano, los animales domésticos y los modelos de mamíferos utilizados en los ensayos de toxicidad. Sin embargo, la información toxicológica relacionada con los animales naturales, plantas y microorganismos en condiciones naturales sigue siendo escasa. [4]. Los ensayos de toxicidad clásicos comúnmente involucran la administración de determinada sustancia a una población de una especie seleccionada bajo condiciones controladas. Esta población, por lo general, se encuentra controlada y aislada de posibles interacciones con otros organismos y sustancias químicas. En estas condiciones los ensayos son repetibles y por tanto científicamente aceptados. En contraposición, los estudios ecotoxicológicos se hacen más complejos y requieren una rigurosa estandarización ya que el ambiente natural es altamente variable y casi imposible de reproducir en condiciones de laboratorio. [5].

Para evaluar el riesgo que una sustancia impone en el ambiente acuático se considera, entre otras, la toxicidad en las concentraciones ambientales. Según Newman [2], la predicción de la toxicidad producida por los contaminantes en un medio acuático es muy compleja. El efecto de la toxicidad dependerá de la agresividad del substrato (composición química, dispersión en el medio, concentración, efectos sinérgicos o antagónicos 
de contaminantes asociados, entre otros), de la resistencia puesta por el ser vivo (asimilación, acumulación, inhibición, muerte, entre otros) y de las características del medio acuático $(\mathrm{pH}$, temperatura, concentración de oxígeno y dióxido de carbono disueltos, sólidos totales disueltos, entre otros).

En función de las limitaciones que los análisis fisicoquímicos imponen, las pruebas de toxicidad con organismos acuáticos y terrestres han sido utilizadas en países altamente industrializados como Alemania, Canadá, Francia y Estados Unidos. A través de estas pruebas se pueden establecer patrones de emisión descriptivos que permitan identificar problemas de lanzamiento de mezclas de sustancias tóxicas, establecer prioridades de control en regiones críticas y acciones correctivas apropiadas, como monitorear un ecosistema acuático teniendo en cuenta los usos preponderantes de las aguas. [6].

Las plantas terrestres son el blanco de las fumigaciones y por lo tanto más vulnerables a la acción directa intencional o no de los plaguicidas. Cedergreen y colaboradores [7] plantearon que debe otorgársele una prioridad mayor a la evaluación del riesgo en estas plantas pues actualmente la utilización sólo se sugiere. Ha sido demostrado en varios experimentos muy recientes que el valor de la extrapolación de los ensayos con plantas acuáticas a plantas terrestres es muy limitado. [8], [9], [10]. Dentro de este contexto, se han identificado plantas terrestres como bioindicadoras de la presencia de tóxicos tanto en aguas como en suelos y se han constituido como centinelas de contaminantes en estos medios. La OECD (Guidelines for the Testing of Chemicals) [11], establece las especies bioindicadoras para el desarrollo de pruebas de toxicidad en los diferentes protocolos de aplicación en ecotoxicologia. Dentro de estas especies se encuentran la lechuga (Lactuca sativa $L$ ) y la cebolla (Allium cepa $L$ ).

El hipoclorito de sodio se utiliza a gran escala. Por ejemplo en la agricultura, industrias químicas, pinturas, industrias de alimentación, industrias del cristal, papeleras y farmacéuticas, industrias sintéticas e industrias de disposición de residuos.

La desinfección tiene por finalidad la destrucción de microorganismos nocivos a la salud, para el control de infecciones y enfermedades. La acción del Hipoclorito de sodio se debe esencialmente a la liberación de Cloro activo. El mecanismo de acción consiste en la inhibición de la reacción enzimática en el interior de la célula y produce desnaturalización e inactivación del ácido nucléico. El Cloro activo liberado actúa sobre las proteínas formando cloraminas que son solubles en agua.

En la industria textil, se utiliza el hipoclorito de sodio como blanqueante, también se puede añadir a aguas residuales industriales para la eliminación de olores. El hipoclorito neutraliza el gas de sulfuro de hidrógeno (H2S) y amonio (NH3). También se puede utilizar para la detoxificación de baños de cianido en industrias del metal. Así mismo, se puede utilizar para la prevención de la formación de algas y crecimiento biológico en torres de enfriamiento, en las plantas de potabilización es utilizado como desinfectante del agua; en las casas, el hipoclorito se usa frecuentemente para la purificación y desinfección doméstica. [12].

Diferentes autores han reportado los efectos genéticos de los oxidantes, como el hipoclorito de sodio, que se presentan en ensayos de toxicidad con plantas y animales. El informe técnico de la AISE, 1997 [13], reconoce la toxicidad del $\mathrm{NaClO}$ en pruebas de ecotoxicidad realizadas en organismos acuáticos, como muy tóxico (CL50<1mg/l). Establece, además, que para especies muy sensibles o en estados inmaduros como en huevo o neonatos, efectos adversos se detectaron a niveles de $1 \mu \mathrm{g}$ $\mathrm{NaClO} / \mathrm{I}$. No descartan, en este informe, la posibilidad de la generación de compuestos organohalogenados (Trihalometanos - THM) por la presencia de desinfectantes clorinados en las aguas residuales domésticas. Investigaciones posteriores realizadas por Monarca y colaboradores [14], confirman la generación de compuestos clorinados (AOX) al incrementar el $\mathrm{NaClO}$ en las dosis evaluadas. Los investigadores detectaron por análisis CG/SM la presencia de THM genotóxicos.

Buschini [15], establecen que el hipoclorito de sodio no está identificado como cancerígeno, sin embargo es su investigación confirman efectos aberrantes sobre cromosomas en Saccharomyces cerevisiae a concentraciones de hipoclorito de sodio reconocidas de desinfección (1-2 ppm). Por otro lado, Monarca [14], confirman lo concluido por Buschini [15], respecto al efecto genotóxico del hipoclorito de sodio, en plantas superiores como en las células radiculares de la haba Vicia faba, a partir de muestras de agua potable desinfectada con diferentes agentes oxidantes. 
Grant [16] y más tarde Saleh [17] concluyen en su investigación que los bulbos de cebolla son un excelente bioindicador para la evaluación de la calidad aguas naturales y residuales domésticas, ya que son muy sensibles a la contaminación y se constituyen como una planta idónea para el diagnóstico de ensayos genotóxicos por su respuesta a daños y aberraciones cromosómicas.

Monte-Egito [18], determinan que compuestos clorados junto con otros componentes de las aguas naturales que reciben vertimientos de pesticidas, metales pesados o aguas servidas domésticas, pueden ser los responsables de la genotoxicidad de las aguas superficiales. Estas técnicas, concluyen los autores, definitivamente se deben implementar a la hora de evaluar sistemas lóticos para uso potable ya que la fisicoquímica no es capaz de establecer los riesgos toxicológicos y la dinámica química de estos compuestos.

\section{METODOLOGÍA}

Cebolla: Cuando un bulbo de cebolla (Allium sp) se rehidrata, se produce una estimulación del crecimiento de las células, lo cual permite la elongación de las raíces de la planta. Sin embargo, cuando la hidratación se lleva a cabo en presencia de sustancias tóxicas, la división celular de los meristemas radiculares puede inhibirse, ya sea retardando el proceso de mitosis o destruyendo las células. Este tipo de alteraciones generalmente impide el crecimiento normal de la raíz y, por tanto, su elongación. [19].

El efecto puede determinarse en forma indirecta, mediante la comparación de la elongación de las raíces de cebollas expuestas al tóxico con las de cebollas no expuestas, luego de un periodo de $72 \mathrm{~h}$ de prueba. La cuantificación del efecto se realiza estableciendo el porcentaje de inhibición del crecimiento de las raíces respecto a la longitud promedio de las raíces del control.

Lechuga: El bioensayo de toxicidad con semillas de lechuga (Lactuca sativa $L$ ) es una prueba estática de toxicidad aguda (120 h de exposición) en la que se pueden evaluar los efectos fitotóxicos de compuestos puros o de mezclas complejas en el proceso de germinación de las semillas y en el desarrollo de las plántulas durante los primeros días de crecimiento.

Como puntos finales para la evaluación de los efectos fitotóxicos, se determina la inhibición en la germinación y la inhibición en la elongación de la radícula y del hipocótilo. Es importante destacar que durante el periodo de germinación y los primeros días de desarrollo de la plántula ocurren numerosos procesos fisiológicos en los que la presencia de una sustancia tóxica puede interferir alterando la supervivencia y el desarrollo normal de la planta, siendo por lo tanto una etapa de gran sensibilidad frente a factores externos adversos. Por otra parte, muchas de las reacciones y procesos involucrados son generales para la gran mayoría de las semillas, por lo que la respuesta de esta especie y los datos obtenidos a partir de la aplicación de esta prueba son en gran medida representativos de los efectos en semillas o plántulas en general.

El éxito o aptitud de una plántula para establecerse en un ambiente determinado es relevante para garantizar la supervivencia de la especie. La evaluación del desarrollo de la radícula y del hipocótilo constituyen indicadores representativos para determinar la capacidad de establecimiento y desarrollo de la planta. [19].

\section{RESULTADOS}

Para evaluar la toxicidad en bioindicadores de origen vegetal, se deben evaluar los cambios evidentes en su desarrollo fisiológico al final de la exposición con la sustancia a evaluar y compararlo con un montaje de control, en las que los organismos no están expuestos a la sustancia de interés y este resultado establece las condiciones de desarrollo normal para comparar con los organismos que están expuestos a la sustancia tóxica.

Para Lactuca sativa, el factor de evaluación que se considera es el desarrollo de la raíz (longitud) y del embrión, llamado también hipocótilo (longitud y coloración). En Allium cepa, se evalúa la longitud de sus raíces y los cambios mitóticos que presentan las células de las raíces en su proceso de desarrollo.

En la tabla I, se muestra los valores promedio de la longitud de las raíces que se obtuvieron en ambas pruebas desarrolladas. Los resultados reportados indican el porcentaje de inhibición de la longitud de las raíces comparado con las longitudes de los recipientes de control (sin sustancia tóxica); así, la Cl50 indica la concentración a la que inhibe el 50\% del crecimiento de la raíz comparado con los valores del control. 
TABLA I

PORCENTAJE DE LONGITUD DE LABL RAİ́CES EN Lactuca sativa Y Allium cepa RESPECTO AL CONTROL, OBTENIDAS EN LA EXPOSICIÓN CON HIPO CLORITO DE SODIO (NaCIO).

\begin{tabular}{|c|c|c|c|c|}
\hline \multirow{2}{*}{$\begin{array}{c}\text { Concentración } \\
(\mathrm{ppm})\end{array}$} & \multicolumn{2}{|c|}{$\begin{array}{c}\text { Lechuga } \\
\text { (Lactuca sativa) }\end{array}$} & \multicolumn{2}{c|}{$\begin{array}{c}\text { Cebolla } \\
\text { (Allium cepa) }\end{array}$} \\
\hline 250 & Raíz (cm) & $\%$ & Raíz (cm) & $\%$ \\
175 & 0 & $0,0 \%$ & 0 & $0,0 \%$ \\
50 & 0,9 & $35,5 \%$ & 0 & $0,0 \%$ \\
25 & 1,0 & $38,1 \%$ & 0 & $0,0 \%$ \\
5 & 1,8 & $68,3 \%$ & 1,1 & $37,0 \%$ \\
\hline Control & 2,6 & $100 \%$ & 3,0 & $100 \%$ \\
\hline
\end{tabular}

Fuente: Autores del proyecto

En esta, se observa que para Lactuca sativa como para Allium cepa hay una total inhibición de germinación a partir de los 250 ppm de Na$\mathrm{ClO}$, pudiéndose asegurar que a esta concentración el Hipoclorito de Sodio es un tóxico letal para estas dos especies.

La columna de (\%) que aparece en la tabla I indica el porcentaje de inhibición de elongación de raíz con respecto a los valores del control. Para ambas especies, se presentaron porcentajes de inhibición de longitud de las raíces, ya que para los hipocótilos de Lactuca sativa no hubo inhibición de desarrollo a este nivel y para Allium cepa, no se considera este valor a nivel de hipocótilo, solo a nivel de raíz. [20].

Para la interpretación del análisis de este porcentaje, se evalúa a que concentración de la sustancia se presenta el $50 \%$ de inhibición en el crecimiento de la raíz o del hipocótilo (para Lactuca sativa) respecto a los valores obtenidos en el control. En los resultados de la tabla 1, se observa que para la raíz de Lactuca sativa este valor de concentración se encuentra entre 5 y 25 ppm de $\mathrm{NaClO}$ y para Allium cepa, es inferior a 5 ppm. Para establecer la concentración exacta en el que se presenta la inhibición del $50 \%$ de la elongación de la raíz de Lactuca sativa, se corrió el estadístico probit con los datos de la tabla 1 , obteniéndose la curva dosis-respuesta de la figura 1. Al extrapolar el $50 \%$ de inhibición sobre las ordenadas, el resultado indica que la concentración inhibitoria (Cl50) fue de 17,27 mgNaClO/I (Log10 1,2373) con un $p<0,05$ (figura I).
FIG. I. CURVA DOSIS-RESPUESTA DE LA INHIBICIÓN DEL NACLO EN LA LONGITUD DE LA RAÍZ DE LACTUCA SATIVA

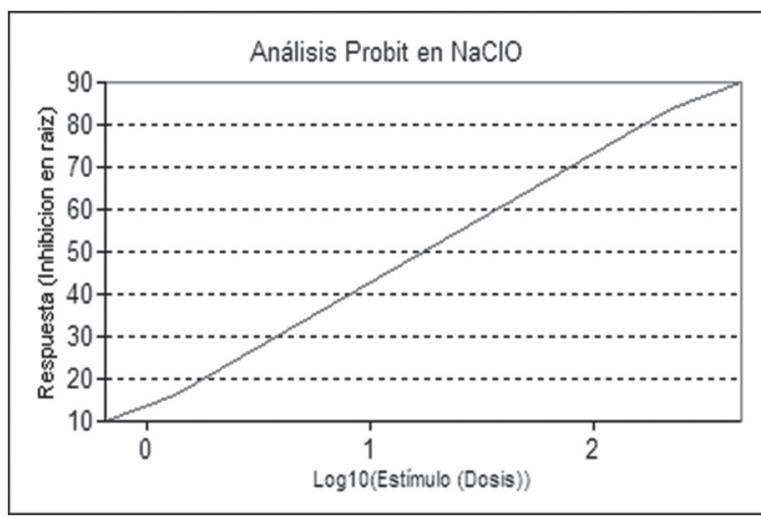

Fuente: Autores del proyecto

Para el caso de Allium cepa, la tabla 1 indica que la Cl50 está a una concentración inferior a 5 ppm, es decir, sería necesario realizar montajes a menor concentración para establecer el valor de la Cl50 para esta especie. Sin embargo, una evaluación de respuesta adicional al utilizar Allium cepa es la valoración de las fases mitóticas. [21], [22], [23]. En la figura II, se muestran los resultados de aberraciones cromosómicas observadas en las células meristemáticas de la raíz de A. cepa a una concentración de 5 ppm de NaClO (la más baja concentración que A. cepa manifestó elongación de la raíces como respuesta).

FIG. II. ABERRACIONES CROMOSÓMICAS PRESENTES EN LAS CÉLULAS MERISTEMÁTICAS DE ALLIUM CEPA EXPUESTAS A NACLO.
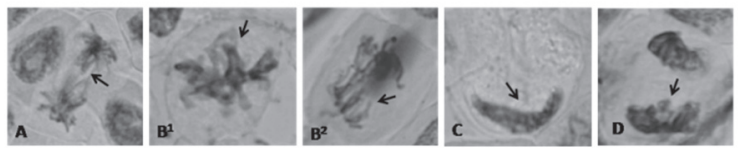

Fuente: Autores del proyecto

Se pueden identificar aberraciones como anafase con puente $(A)$, rompimiento de cromosomas en metafase (B1, B2), malformación de células con núcleos periféricos (C) y telofase con pérdida de cromosomas (D) (Figura 2). Estas aberraciones han sido reportadas e identificadas por varios autores, las cuales han sido compiladas por Morais \& Marin. [24].

\section{DISCUSIÓN}

La degradación del Hipoclorito de sodio en el ambiente es lenta y depende de las condiciones del medio, como la concentración inicial de $\mathrm{NaClO}$, 
el $\mathrm{pH}$, la radiación UV y la presencia de metales pesados, quienes actúan como catalizadores. El proceso de degradación termina cuando los cloritos y cloratos llegan a oxígeno. [25].

Los resultados encontrados en esta investigación demuestran la toxicidad en organismos vegetales, siendo $\mathrm{Cl} 50$ de 17.27 ppm para Lactuca sativa e inferior a $5 \mathrm{ppm}$ para Allium cepa, con el agravante adicional de generar un efecto genotóxico en las células meristemáticas de la raíz de la cebolla a esta misma concentración. Se identificaron aberraciones cromosómicas en prácticamente todas las fases mitóticas (Figura II).

Revisando la información de uso de un producto comercial, recomiendan aplicar $1 / 4$ de taza por cada 4 litros para despercudir la ropa, y si se quiere para desinfectar los pisos, aplicar $3 / 4$ de taza en los mismos 4 litros de agua jabonosa. Para el primer caso, considerando un volumen estandarizado de $236.6 \mathrm{ml}$ por taza y a partir de una concentración en dilución de $5.5 \%$ de $\mathrm{NaClO}$ (recomendado y la presentación comercial para uso doméstico), se tendría una concentración aproximada de 750 ppm de $\mathrm{NaClO}$. Para el segundo uso, teniendo en cuenta las mismas consideraciones, se tendría una concentración de 2250 ppm. Ambas concentraciones están fuera del contexto de protección ambiental, teniendo en cuenta las concentraciones inhibitorias $(\mathrm{Cl} 50)$ reportadas en esta investigación utilizando Lactuca sativa y Allium cepa de 17.27 ppm y de 5 ppm, respectivamente. Si se consideran adicionalmente los registros internacionales para animales acuáticos, las concentraciones letales (CL50) que reporta la Merck, [26] son:

Onchorhynchus mykiss CL50: 0,07 mg NaClO/l /48h Pimephales promelas CL50: 1.34 mg NaClO/I /96h Lepomis macrochirus CL50: $1.1 \mathrm{mg} \mathrm{NaClO/I/96} \mathrm{h}$ Daphnia magna CE50: 0.07 - 0,7 mg NaClO/I /24 h

Seguramente se están envenenando hace mucho tiempo los organismos acuáticos por los vertidos de cientos de casas que utilizan a diario limpiadores como el $\mathrm{NaClO}$ en sus actividades domésticas sin ningún control ni restricción. Aunque es evidente el efecto que tiene el hipoclorito sobre los ecosistemas, es importante también resaltar la aparición de compuestos organohalogenados trihalometanos-, los cuales se dan por la reacción química entre el cloro y la materia orgánica presentes en las aguas naturales o residuales.

El cloro que se vierte a las aguas como desinfectante o limpiador en los productos de aseo reacciona con los compuestos orgánicos sustituyendo tres de los cuatro átomos de hidrogeno de las moléculas de metano por átomos de halógenos, formándose trihalometanos, nuevos compuestos como cloroformo, dibromoclorometano, bromoformo y bromodiclorometano, los cuales son potenciales cancerígenos. De acuerdo con la organización mundial de la salud clasifica el cloroformo y el bromodiclorometano como posibles carcinógenos para las personas en ciertas condiciones de exposición.

Por otra parte, diferentes estudios epidemiológicos han hallado asociación entre el riesgo de cáncer de vejiga a largas exposiciones de los trihalometanos (más de 30 años) [27]. Algunos estudios en humanos muestran resultados concordantes con la relación descrita en animales entre exposición a trihalometanos y tumores en el hígado y riñón [28]. La exposición a estos compuestos es determinada por la concentracion de hipoclorito de sodio emitida al ambiente.

Los estudios que se han realizado para determinar el grado de toxicidad por parte de los THM en los países europeos, en Latinoamérica y en especial en Colombia, no se reportan análisis de parámetros de compuestos precursores que permitan determinar concentraciones de THM en las cuencas hidrográficas que reciben las aguas servidas de las ciudades, mucho menos la cantidad de estos que llegan a las aguas potables. En este estudio, se plantea la posibilidad de riesgo de la presencia de estos compuestos en las aguas de los ríos colombianos.

Finalmente se plantea la incidencia de limpiadores y desinfectantes como potenciales contaminantes al medio debido a la presencia de hipoclorito de sodio mal dosificado domésticamente y a su posterior reacción con el entorno. De acuerdo con los resultados obtenidos se observa que el Allium cepa presenta mayor sensibilidad a diferentes concentraciones de hipoclorito con respecto a la Lactuca Sativa, por lo que se propone el Allium cepa como un bioindicador determinante de la genotoxicidad de sustancias químicas vertidas en ríos y la calidad de la misma. 


\section{CONCLUSIONES}

La Cl50 del hipoclorito de sodio al 5\% (versión comercial para uso doméstico) es de 17.27 ppm para Lactuca sativa e inferior a 5 ppm para Allium cepa. Estas concentraciones son muy inferiores a las dosis que se manejan para uso doméstico. Haciendo un cálculo a partir de las recomendaciones del mismo fabricante, se utiliza una concentración aproximada de $750 \mathrm{ppm}$ de $\mathrm{NaClO}$ para despercudir la ropa y de 2250 ppm para desinfectar pisos.

Se identificaron las aberraciones cromosómicas de anafase con puente, rompimiento de cromosomas en metafase, malformación de células con núcleos periféricos y telofase con pérdida de cromosomas, en prácticamente todas las fases mitóticas, demostrando la acción genotóxica de este compuesto a partir de concentraciones de 5 ppm, en células meristemáticas de Allium cepa.

Los resultados permiten establecer que Allium cepa presenta mayor sensibilidad a diferentes concentraciones de hipoclorito con respecto a la Lactuca sativa, por lo que se propone el uso de A. cepa como un bioindicador determinante de la genotoxicidad de sustancias químicas vertidas en ríos y para uso en la evaluación de la calidad de cuerpos de agua naturales y receptores de vertimientos residuales.

Debido a la incidencia de los limpiadores y desinfectantes como potenciales contaminantes al medio acuático, y a la presencia de hipoclorito de sodio y a su posterior reacción con el entorno, se recomienda implementar programas de monitoreo de compuestos organohalogenados (trihalometanos) en aguas naturales y cuerpos receptores de vertimientos, parámetro que tradicionalmente se evalúa únicamente en aguas potables.

\section{AGRADECIMIENTOS}

Los autores agradecen a la Facultad de Química Ambiental y al Grupo de Investigaciones Ambientales para el Desarrollo Sostenible - GIADS de la Universidad Santo Tomás - Bucaramanga.

\section{REFERENCIAS}

[1] Relyea, R., Hoverman, J. "Assessing the ecology in ecotoxicology: a review and synthesis in freshwater systems". Ecology Letters. 9; 1157-71. 2006.
[2] Newman, M. "Fundamentals of Ecotoxicology". 2nd Ed. Lewis Publishers. CRC Press, Inc., Boca Ratón, Fl, pp. 1-250. 2001.

[3] Kruijf, H. A. M. What is Ecotoxicology. In: Kruijf, H. A. M.; Zwart, D.; Viswanathan, P.N. \& Ray, P.K. (eds). Manual on aquatic ecotoxicology. Oxford: Klumer Academic Publishewrs, 1988. p: 18-21.

[4] Hayes, T., Case, P., Chui, S., Chung, D., Haeffele, C., Haston, K. "Pesticide mixtures, endocrine disruption, and amphibian declines: are we underestimating the impact?" Environ. Health Perspect., 114(1); 40-50. 2006.

[5] Fleeger, J., Carman, K., Nisbet, R. "Indirect effects of contaminants in aquatic ecosystems". Sci. Total Environ., 317; 207-33. 2003.

[6] Kummerer, K. "Drugs, diagnostic agents and disinfectants in waste water and water-a review". Chemosphere. 45; 957-69. 2001.

[7] Cedergreen, N., Kudsk, P., Mathiassen, S., Streibig, J. "Combination effects of herbicides on plants and algae: do species and test systems matter?". Pest. Manag. Sci., 63; 282-95. 2007.

[8] Sørensen H., Cedergreen, N., Skovgaard, I., Streibig, J. "An isobole-based statistical model and test for synergism/antagonism in binary mixture toxicity experiments". Environ. Ecol. Stat., 14, in press. 2007.

[9] Cedergreen, N., Kamper, A., Streibig J. "Is prochloraz a potent synergist across species? A study on bacteria, daphnia, algae and higher plants". Aquat. Toxicol., 78; 243-52. 2006.

[10] Cedergreen, N., Kudsk, P., Mathiassen, S.K., Sørensen, H., Streibig, J. The reproducability of binary mixture toxicity studies. Environ.Toxicol. Chem. 26 (1); 149-56. 2007.

[11] OECD. GUIDELINE FOR THE TESTING OF CHEMICALS PROPOSAL FOR UPDATING GUIDELINE 208. Terrestrial Plant Test: 208: Seedling Emergence and Seedling Growth Test. DRAFT DOCUMENT. September 2003.

[12] Water Treatment Solutions- Lenntech. "Desinfectantes: Hipoclorito de sodio". Disponible on line: http://www.lenntech.es/procesos/desinfeccion/quimica/desinfectantes-hipoclorito-desodio.htm.

[13] AISE. Association Internationale de la Savonnerie, de la Detergence el des Produits d'Entretien. 
Technical Task Force HYPOCLORITE. December, 13 pp. 1997.

[14] Monarca S, Rizzoni M, Gustavino B, Zani C, Alberti A, Feretti D, Zerbini I. "Genotoxicity of surface water treated with different disinfectants using in situ plant tests". Department of Hygiene and Public Health, University of Perugia, Italy. 2003.

[15] Buschini, A., Carboni, P. M., Paola, F. P. \& Rossi, C. "Sodium hypochlorite-, chlorine dioxide- and peracetic acid-induced genotoxicity detected by the Comet assay and Saccharomyces cerevisiae D7 tests". Mutagenesis, 19 (2):157-162. 2004.

[16] Grant, W.F. "Chromosome Aberrations in Plants as a Monitoring System". Environ. Health Perspect., 27(12): 37-43. 1978.

[17] Saleh, K., Khanfar, A. \& Mosaad, I. "Detection of abnormal and micronucleus in root tips of Allium cepa cultivated in water wells". Inter. J. App. Environ. Sciences, (Sept): 4pp. 2009.

[18] Monte-Egito, L. C., Medeiros, M., Batistuzzo de Medeiros, S. R. \& Agnez-Lima, L. F. "Cytotoxic and genotoxic potential of surface water from the Pitimbu river, northeastern/RN Brazil". Gen. \& Mol. Biol., 30 (2): 435-441. 2007.

[19] Castillo, G (ed.). "Ensayos toxicológicos y métodos de evaluación de calidad de aguas. Estandarización, intercalibración, resultados y aplicaciones". IDRC, IMTA, Canadá. 202 pp. 2004.

[20] Rank, J. \& Nielsen, M.H. "Evaluation of Allium anaphase-telophase test in relation to genotoxicity screening of industrial wastewater". Mut. Res., 312(1994):17-24. 1994.

[21] Fiskesjö, G. "The Allium Test as Standard in Environmental Monitoring". Hereditas, 102: 99-112. 1985.

[22] Fiskesjö, G. "The Allium Test in Wastewater Monitoring". Environ. Toxicol. and Water Quality 8: 291-298. 1993.

[23] Fiskesjö, G. "Allium Test for Screening Chemicals; Evaluation of Cytological Parameters". En: W. Wancheng, J. W. Gorsuch y J. S. Hughes (eds.). Plants for Environmental Studies. CRC. 1997.

[24] Morais-Leme, D. \& Marin-Morales, M.A. "Allium cepa test in environmental monitoring: A review on its application". Mutation Res., 682(2009): 71-81. 2009.
[25] BLACK \& VEATCH CORPORATION. White's Handbook of Chlorination and Alternative Desinfectants. 5th Edition. John Wiley \& Songs, Inc., Publication.1009, pp. 2010.

[26] Merck Catalogue. ChemDAT - The Merck Chemical Database. 2000.

[27] García-Sotillo, M.F. “Efectos de la Cloración de efluentes cloacales tratados sobre la calidad del agua de ambientes loticos naturales" Trabajo Monográfico para optar el título de Licenciada en Ciencias Biológicas. Universidad Nacional de la Patagonia San Juan Bosco. Facultad de Ciencias Naturales. 63 pp. 2011.

[28] Howe, G. K., Jacobs, M. \& Clapp, R. W. "Causas Ambientales y Ocupacionales del Cáncer: revisión de la literatura científica reciente. Centro Lowell para la Producción Sostenible. Universidad de Massachusetts Lowell. 49 pp. 2005. 